Relations industrielles

Industrial Relations

\title{
The Unemployed : A Social-Psychological Portrait, by D.W. Tiffany, J.R. Cowan and P.M. Tiffany, New Jersey, Prentice-Hall Inc., 1970, $180 \mathrm{pp}$.
}

\section{Jean Sexton}

Volume 26, numéro 3, 1971

URI : https://id.erudit.org/iderudit/028259ar

DOI : https://doi.org/10.7202/028259ar

Aller au sommaire du numéro

Éditeur(s)

Département des relations industrielles de l'Université Laval

ISSN

0034-379X (imprimé)

1703-8138 (numérique)

Découvrir la revue

Citer ce compte rendu

Sexton, J. (1971). Compte rendu de [The Unemployed : A Social-Psychological Portrait, by D.W. Tiffany, J.R. Cowan and P.M. Tiffany, New Jersey,

Prentice-Hall Inc., 1970, 180 pp.] Relations industrielles / Industrial Relations, 26(3), 781-782. https://doi.org/10.7202/028259ar

Tous droits réservés @ Département des relations industrielles de l'Université Laval, 1971
Ce document est protégé par la loi sur le droit d'auteur. L’utilisation des services d’Érudit (y compris la reproduction) est assujettie à sa politique d'utilisation que vous pouvez consulter en ligne.

https://apropos.erudit.org/fr/usagers/politique-dutilisation/ 
Du côté syndical, même observation. Les syndicats, jusqu'ici, ont été assez peu démocratiques, précisément à cause de la faible participation des travailleurs. Un changement notable est en train de se produire. Ou le nouveau type d'ouvrier exigera de participer davantage à la vie syndicale, ou le syndicalisme en viendra à craquer. Le phénomène est apparent déjà dans la volonté de plus en plus marquée des travailleurs de régler leurs problèmes au niveau de la base.

On remarquera une tendance analogue dans les autres secteurs de l'activité économique, sociale et politique. Les Auteurs expriment bien ce nouvel état, lorsqu'ils écrivent: «Beaucoup de travailleurs vivent aujourd'hui dans l'aisance et une proportion de plus en plus forte de jeunes sont instruits. L'ouvrier de type nouveau apporte à de vieilles institutions des capacités nouvelles et des standards nouveaux de compétence. Il a une orientation très moderne, un sens différent de l'égo et une attitude beaucoup plus égalitaire et libérale au sujet de scs réactions et de ses responsabilités à l'égard d'autrui. Il n'accepte pas le système qui a été conçu pour le travailleur traditionnel. La fragmentation et la monotonie du travail lui apparaissent répugnantes et dépourvues de sens. Bref, pour l'ouvrier moderne, le travail n'est devenu qu'un moyen d'accroître son niveau de vie $\gg$.

En réalité, on peut dire que le travailleur est en voie d'être transfiguré par l'accroissement de la richesse et l'instruction, de telle manière qu'il veut et peut jouer un rôle différent dans son milieu de travail, dans son syndicat, dans la société. Cependant, étant donné que les institutions restent toujours adaptées à la mentalité des ouvriers traditionnels, il n'y a aucune possibilité pour l'ouvrier moderne de tenir ce rôle nouveau. Même dans les usines les plus automatisées, cette question n'est pas résolue, parce que, même si l'ouvrier se rend compte qu'il possède plus de responsabilités, il ne sent pas qu'il puisse obtenir un statut équivalent à son mérite. En conséquence, est-il frustré et mécontent, révolté quand il le peut, aliéné, s'il ne le peut pas. Et c'est ceci qui engendre les tensions qui conduisent à une détérioration considérable de la vie sociale. Pour corriger cet état de choses, quelles peuvent être les solutions valables?
Les Auteurs, dans ce qu'on peut considérer comme le troisième volet du triptyque, n'ont pas voulu élaborer. Ils n'offrent que quelques idées générales: mettre de côté ce qu'ils estiment être la philosophie désuète d'une organisation socio-économique fondée sur l'idée de rareté, alors qu'il y a surabondance de biens, pour adopter un comportement nouveau qui rejette les notions de concurrence et de consommation; faciliter aux travailleurs l'accès à la vraie liberté et à l'exercice de la responsabilité dont on sait que les applications concrètes à tout niveau peuvent être quasi infinies. Ces deux seuls faits, et il y en a combien d'autres, ouvrent très grande la porte à ceux qui sont encore capables d'observation, d'imagination créatrice.

《The Emerging Worker» n'est pas qu'un ouvrage instructif décrivant de très près une situation sociale en voie d'évolution rapide dans les pays qui ent atteint le stade de la société d'abondance. Les Auteurs ont beaucoup lu, beaucoup étudié, beaucoup observé. C'est pourquoi pareille étude s'impose comme sujet de méditation à ceux qui sont engagés dans l'action collective, y assumant à un degré quelconque des responsabilités de gouvernement et de direction.

\section{André ROY}

\section{The Unemployed : A Social-Psychological} Portrait, by D.W. Tiffany, J.R. Cowan and P.M. Tiffany, New Jersey, Prentice-Hall Inc., 1970, 180 pp.

Voici un ouvrage qui envisage le problème du chômage d'une façon entièrement différente de celles qu'on connaît habituellement. Au lieu de s'attarder à l'examen de l'aspect économique des sans-emplois, les Auteurs ont choisi de considérer les aspects psychologiques de ce problème. Le but principal de cet ouvrage est d'étudier les caractéristiques socio-psychologiques qui, reliées avec les obstacles de l'environnement, produisent le chômage.

En guise de conclusion principale, les Auteurs croient qu'on devrait traiter le chômage à la fois sous ses aspects macroéconomiques et, avec autant d'importance, sous ses aspects psychologiques.

Il ne s'agit aucunement d'une étude de cas. En fait, ce livre n'est qu'un résumé de la littérature sur le sujet. Afin d'illustrer les principes qu'ils émettent, les 
Auteurs insistent sur les programmes de formation professionnelle en vigueur aux Etats-Unis. Ils y notent les trois modèles selon lesquels on peut élaborer ces prugrammes et concluent, par leur modèle socio-psychologique, qu'un nombre important de clients des programmes de réhabilitation professionnelle, des programmes de main-d'œuvre et des bureaux de placement sont de facto des cas problèmes.

Les Auteurs présentent la psychologie du chômeur en cinq parties. Après un rappel de la façon traditionnelle d'aborder le problème des sans-emplois, on retrouve l'examen du chômage comme cause de problèmes psychologiques, et l'examen des problèmes psychologiques comme cause du chômage. Après ce tour d'horizon assez superficiel, les Auteurs évaluent les programmes de réhabilitation professionnelle, avancent un certain nombre de recommandations et définissent des domaines de recherche pour l'avenir.

Il n'y a aucun doute que l'approche utilisée par ces trois Auteurs pour étudier le problème du chômage est intéressante. L'examen méthodique des facteurs autres que les traditionnelles causes économiques s'avère de plus en plus nécessaire en ce domaine. Cependant, ce n'est pas dans ce livre que nous pouvons trouver une anproche rigoureuse pour satisfaire ce besoin. Les Auteurs ont le mérite de poser une question extrêmement difficile à envisager, mais la réponse qu'ils fournissent ne peut être complètement acceptée à cause d'un certain nombre de limites qu'on peut noter dans leur étude.

La première difficulté qu'on peut identifier dans cet ouvrage est d'ordre méthodologique. Les Auteurs notent que leur étude est le fruit de cinq années ie recherches et d'enquêtes. Cependant, ils ne fournissent au lecteur ni le cadre de référence qui les a guidés, ni les hypothèses de travail qu'ils ont dû faire, ni les limites qu'eux-mêmes voyaient à l'intérieur de leur propre recherche. En plus, il n'est pas rare de retrouver dans cet ouvrage des affirmations gratuites qui auraient pu être appuyées par des vérifications empiriques.

En second lieu on ne retrouve dans cet ouvrage aucune preuve empirique des différentes conclusions avancées. Même si a priori il semble logique et nécessaire de considérer les facteurs psychologiques lorsqu'on examine le chômage ; ce n'est pas par cet ouvrage qu'on saura dans quelle mesure ils peuvent expliquer ce phénomène. Nous avons déjà noté que les Auteurs reprochaient à la littérature de ne considérer que l'aspect économique du chômage. Même s'ils admettent que leur approche n'est pas la réponse unique au problème, ils ne mettent pas ce principe en application dans les diagnostics qu'ils posent et les solutions qu'ils avancent. En plus des causes psychologiques et économiques, il est fort probable que le chômage ait aussi des causes sociologiques, juridiques, institutionnelles, etc. Il devient alors prétentieux de vouloir répondre à un problème par une approche unique. L'approche multidisciplinaire peut être plus facile à proposer qu'à effectuer. Mais on ne le saura que lorsqu'elle sera réellement mise à l'épreuve.

Une autre faute des Auteurs serait de ne pas distinguer les différentes sortes de chômage. Mềme si on est d'accord avec leur principe de base, à savoir qu'on ne doit idéalement tolérer aucun chômage, on doit cependant admettre qu'en pratique il existera toujours un certain chômage frictionnel (tel que défini par Lipsey) principalement à cause des imperfections du marché du travail et de l'information incomplète sur ce marché. $\mathrm{Ce}$ dernier problème d'information est une autre lacune dans cet ouvrage. On y fait à peine référence et on oublie qu'il est fort possible que le manque de données au niveau local puisse souvent être la cause d'une mauvaise allocation des ressources humaines dont la principale conséquence est le chômage.

Donc cet ouvrage pose une question intéressante à la réalité, mais les faiblesses déjà notées enlèvent à cette étude l'intérêt qu'il aurait pu avoir eut-il été mieux présenté et structuré.

\section{Jean SEXTON}

\section{Social Psychology of the Work Organ-} ization, by Arnold S. Tannenbaum, Belmont, Wadsworth, Behavioral Science in Industry Series, 1966, $136 \mathrm{pp}$.

Training in Industry: The Management of Learning, by Bernard $M$. Bass and James A. Vaughan, Belmont, BrooksCole, Behavioral Science in Industry Series, 1966, 164 pp.

Les deux volumes que nous présentons sont issus de la même série, sous la direction de V.H. Vroom qui en est 